\title{
Los pilares de las relaciones internacionales de los gobiernos locales de México: el caso de Baja California
}

\author{
RAFAel VeLÁZQuez Flores* \\ ERnESTO AlOnSO LEÓN VALDEZ**
}

Artículo recibido: 11 de mayo de 2015

Artículo aprobado: 5 de agosto de 2015

Doi: dx.doi.org/10.12804/desafios28.1.2016.04

Para citar este artículo: Velázquez Flores, R. \& León Valdez E.A. (2016). Los pilares de las relaciones internacionales de los gobiernos locales de México: El caso de Baja California. Desafíos, 28(I), 165-201. Doi: dx.doi.org/10.12804/desafios28.1.2016.04

\section{Resumen}

El objetivo principal de este articulo es identificar los factores que explican el reciente dinamismo de los gobiernos locales mexicanos en los asuntos externos. El argumento principal es que los cambios recientes en el sistema internacional, los avances democráticos en México, la transformación del modelo de desarrollo económico a mediados de los años ochenta y las nuevas percepciones y preferencias personales de las autoridades gubernamentales son factores que explican un mayor interés de los actores subnacionales

\footnotetext{
* Profesor-investigador en la Facultad de Economía y Relaciones Internacionales (FEYRI) de la Universidad Autónoma de Baja California (UABC). Doctor en Estudios Internacionales de la Universidad de Miami. Presidente de la Asociación Mexicana de Asuntos Internacionales (AMEI) 2015-2017. Miembro del Sistema Nacional de Investigadores (SNI) del CONACYT, México. Correo electrónico: rafael.velazquez@uabc.edu.mx

** Licenciado en Relaciones Internacionales por la Facultad de Economía y Relaciones Internacionales de la Universidad Autónoma de Baja California. Asistente de investigación del proyecto: Calidad de la democracia y administración de justicia en América Latina. Realizó su práctica profesional en la Dirección General de Coordinación Política de la Secretaría de Relaciones Exteriores en el área de gobiernos locales. Correo electrónico: alonso.leon@ uabc.edu.mx
} 
mexicanos para participar en actividades globales. El texto está dividido en cuatro partes. La primera define el concepto de paradiplomacia en perspectiva comparada con la política exterior. La segunda parte desarrolla los pilares que explican el nuevo dinamismo internacional de los gobiernos locales mexicanos. La tercera describe el marco jurídico mexicano que norma las relaciones internacionales de los gobiernos locales. La última parte examina el caso de Baja California, un estado mexicano fronterizo altamente activo en asuntos externos.

Palabras clave: Paradiplomacia, México, Baja California, Marco legal, comercio e inversiones.

\title{
The Pillars of the International Relations of Local Governments in Mexico: The Case of Baja California
}

\begin{abstract}
The key objective of this article is to identify the main factors that explain the growing interest of Mexican local governments in external affairs. The main argument is that recent changes in the international system, domestic democratic developments, the transformation of the economic development model in the 1980s, and new perceptions and personal preferences of governmental authorities are factors that explain the growing interest of subnational actors to participate in global activities. The article is divided into four parts. The first one defines the concept of paradiplomacy and foreign policy. The second part analyzes the pillars that explain the new international dynamism of local Mexican governments. The next section describes the legal framework that regulates the international activity of local governments. Finally, the last part examines the case of Baja California, a border Mexican state that recently has been very active in foreign affairs.
\end{abstract}

Keywords: Paradiplomacy, Mexico, Baja California, legal framework, trade and investment. 


\title{
Os pilares das relações internacionais dos governos locais do México: o caso da Baixa Califórnia
}

\begin{abstract}
Resumo
O objetivo principal deste artigo é identificar os fatores que explicam o recente dinamismo dos governos locais mexicanos nos assuntos externos. O argumento principal é que as mudanças recentes no sistema internacional, os avances democráticos no México, a transformação do modelo de desenvolvimento económico em meio dos anos oitenta e as novas percepções e preferências pessoais das autoridades governamentais são fatores que explicam um maior interesse dos atores subnacionais mexicanos para participar em atividades globais. O texto está dividido em quatro partes. $A$ primeira define o conceito de paradiplomacia em perspectiva comparada com a politica exterior. A segunda parte desenvolve os pilares que explicam o novo dinamismo internacional dos governos locais mexicanos. A terceira descreve o marco jurídico mexicano que norma as relações internacionais dos governos locais. A última parte examina o caso da Baixa Califórnia, um estado mexicano fronteiriço altamente ativo em assuntos externos.
\end{abstract}

Palavras -chave: Paradiplomacia, México, Baixa Califórnia, Marco legal, comércio e investimentos.

\section{Introducción}

La participación de los gobiernos locales mexicanos en asuntos internacionales es un fenómeno relativamente novedoso. Anteriormente, esta actividad estaba bastante limitada y pocos actores subnacionales gozaban de una presencia en tal ámbito. Algunos municipios y estados fronterizos, y los que poseían mayores atractivos turísticos tenían más contactos con el exterior. Esta limitación se debía a que el gobierno federal mantenía prácticamente un monopolio de la política exterior del país. La Constitución mexicana le otorga al poder Ejecutivo amplias facultades en materia de relaciones exteriores y, al mismo tiempo, limita la participación de los gobiernos locales en asuntos internacionales. Por otra parte, el sistema político con un fuerte rasgo presidencialista impedía que los gobernadores o los 
presidentes municipales cuestionaran la autoridad del presidente en la materia. Además, todos los gobernadores y la inmensa mayoría de los presidentes municipales pertenecían a un solo partido; por lo tanto, estos políticos le debían lealtad y disciplina al presidente, quien era del mismo partido.

A finales del siglo XX y principios del XXI, los gobiernos locales empezaron a desarrollar una mayor actividad en el ámbito global. Hoy, este tipo de participación es una realidad concreta y constante. Los estados y municipios de México mantienen una intensa presencia en el exterior y su visibilidad ha aumentado de manera significativa en los últimos años. En este sentido, resulta relevante identificar las causas que explican este notable cambio. Por lo tanto, la pregunta central de este ensayo es: ¿Qué factores explican el incremento de la actividad internacional por parte de los gobiernos locales mexicanos?

En este orden de ideas, el objetivo central de este artículo es identificar las razones que explican el nuevo dinamismo internacional por parte de los actores subnacionales mexicanos. El ensayo parte de dos hipótesis. La primera es que las relaciones internacionales de los gobiernos locales mexicanos han estado determinadas, de manera general, por tres grandes pilares. El primero es el ambiente internacional, es decir, el contexto externo ha sido un factor relevante para que los gobiernos estatales y municipales busquen ahora insertarse con mayor interés en la dinámica internacional. Los elementos más importantes en este nivel son la estructura de poder global y la naturaleza de los actores internacionales. El segundo pilar se ubica en el contexto interno. En otras palabras, la nueva dinámica económica y política al interior del país ha sido también un elemento que ha permitido una mayor participación de los gobiernos locales en las relaciones internacionales. Aquí, las principales variables son el funcionamiento del sistema político y el modelo de desarrollo económico. Finalmente, el tercer pilar es el individuo. Es decir, las características personales de los funcionarios locales también determinan, en parte, la inserción de sus gobiernos en el plano internacional, es decir, si una autoridad considera que esa actividad es relevante, entonces es probable que ese gobierno local tenga una presencia en esos temas. 
Aquí, los elementos que influyen son las ambiciones políticas de las personas, la formación académica y la percepción que el individuo tenga sobre la importancia de los temas internacionales.

La segunda hipótesis es que un cambio importante en cualquiera de estos pilares representará una transformación significativa en la participación de los gobiernos locales mexicanos en relaciones internacionales. Es decir, si el sistema internacional experimenta cambios profundos, si el sistema político mexicano se modifica sustancialmente y se adopta un nuevo modelo de desarrollo económico, y si hay un cambio en las percepciones de los individuos, entonces el tipo y la intensidad de participación de los gobiernos locales en asuntos globales también experimentarán cambios importantes.

Para ilustrar este tema, el ensayo utiliza el caso de Baja California. Este estado mexicano tiene un perfil internacional natural y por ello es importante identificar cómo se posiciona en los asuntos exteriores. Baja California fue la primera entidad que no fue gobernada por el partido político mexicano, el cual duró en el poder prácticamente setenta años. En 1989, el Partido Acción Nacional (PAN) derrotó al Partido Revolucionario Institucional (PRI) en las elecciones para gobernador de ese estado. Por lo tanto, Baja California se convirtió en un activo participante en los asuntos exteriores, puesto que no tenía compromisos directos en esa materia con el gobierno federal.

El artículo está dividido básicamente en cuatro grandes partes. La primera define el concepto de paradiplomacia y de política exterior. La segunda parte explica la participación de los gobiernos locales mexicanos en asuntos exteriores a partir de: a) los rasgos del sistema internacional; b) los elementos internos, como son los cambios en el sistema político y la adopción de un nuevo modelo de desarrollo económico; y c) las percepciones y preferencias individuales. La tercera parte examina el marco legal en el que se sustenta la acción exterior de los gobiernos locales en México, y la cuarta parte, finalmente, desarrolla el caso de Baja California, como un ejemplo de un actor altamente activo en relaciones internacionales. 


\section{Paradiplomacia y política exterior}

En los últimos años han surgido diversos conceptos que buscan definir las actividades de carácter externo de los gobiernos no centrales o subnacionales. "Paradiplomacia" fue uno de los primeros términos utilizados para tal fin. En la literatura de la disciplina de las Relaciones Internacionales, los primeros autores en emplear esa palabra fueron Yvo Duchacek \& Panayotis Soldatos en sus obras: The territorial dimension of politics: Within, among and across nations (1987) y Federalism and international relations: The role of subnational units (1990), respectivamente. Estos trabajos se desarrollaron en un contexto en el que los estados federales, especialmente los de Estados Unidos, empezaron a demandar una mayor participación en los asuntos internacionales luego de una creciente interdependencia económica a finales de la década de los setenta e inicios de la de los años ochenta. Sin embargo, diversos autores han desarrollado una variedad de términos para describir esas actividades; por ejemplo, se han utilizado los términos "diplomacia local", "diplomacia paralela", "diplomacia federativa", "acción exterior de los gobiernos locales", "diplomacia constitutiva”, entre otros. ${ }^{1}$

El concepto surgió debido a las diferencias entre los actores federales y los locales en la década de los años setenta. Como la exclusividad en la política exterior se ubicaba en el nivel federal, entonces los gobiernos estatales empezaron a tener fricciones y diferencias con las autoridades centrales. Ante este conflicto, resultó interesante estudiar la relación entre estos dos niveles de gobierno en materia de política exterior y la actividad internacional desarrollada por las entidades federales de manera independiente.

Para Duchacek \& Soldatos (citado en Aguirre, 2000), la paradiplomacia consiste en los contactos, actividades, procesos e iniciativas externas entre gobiernos no centrales (estados federados, provincias, cantones, länders, etc.) y otros actores internacionales, tales como los Estadonaciones, gobiernos locales similares, empresas privadas, organiza-

\footnotetext{
(2012)

Sobre esta discusión, se recomienda: Zidane Zeraoui (2011) y Adriana Sletza Ortega
} 
ciones internacionales, entre otros. Estas actividades no representan la política exterior de un Estado, puesto que ésta es una atribución exclusiva de los gobiernos centrales, pero se trata de acciones que complementan y apoyan la política exterior, ya que el sufijo "para" significa "paralelo", "junto a" o "asociado".

En este sentido, la paradiplomacia no se ocupa de los temas de la high politics (política alta o de primer nivel) como sería la seguridad nacional, defensa, los tratados de libre comercio, la celebración de alianzas, etc., que son exclusivos del gobierno federal. En cambio, la paradiplomacia se ocupa de la low politics (política baja o de segundo nivel), que incluye temas de protección al medio ambiente, atracción de inversiones, turismo, intercambios culturales, entre otros.

A diferencia de la paradiplomacia, la política exterior es el conjunto de acciones que tiene un Estado más allá de sus fronteras en función del interés nacional. En este contexto, solamente los Estados representados por los gobiernos federales pueden elaborar "política exterior". En el marco del Derecho Internacional, solamente los Estados son responsables de los actos que se desplieguen en el exterior. Por lo tanto, los gobiernos locales solamente pueden desarrollar "relaciones internacionales", es decir, pueden establecer contactos con pares, organizaciones internacionales, empresas, funcionarios diplomáticos, y con otros Estados a efecto de promover sus propios intereses, pero estoo no significa que sea la política exterior del país al que pertenecen. Sin embargo, estos actores locales pueden buscar incidir en las decisiones de los gobiernos federales en materia de política para que se tomen decisiones en favor de sus comunidades.

\section{Los pilares que explican el nuevo dinamismo de los gobiernos locales mexicanos en asuntos exteriores}

\subsection{El primer pilar: el ambiente internacional y la naturaleza de los actores externos}

Desde la perspectiva del primer pilar, los recientes cambios en el sistema internacional explican el aumento de actividades externas 
de los gobiernos locales mexicanos. Por un lado, el fin de la Guerra Fría representó un importante cambio en el equilibrio del poder del sistema internacional. La desaparición de la URSS y del bloque socialista modificó la correlación de fuerzas en el contexto internacional, mientras Estados Unidos se establecía como la superpotencia económicamilitar. Pero otros actores empezaron a competir fuertemente con este último país en el ámbito económico, como la Unión Europea, Japón y China. Entonces, el sistema internacional contemporáneo está caracterizado por una unipolaridad desde el punto de vista militar y una multipolaridad en la esfera económica.

Con el fin de la Guerra Fría, los objetivos de política exterior de los principales actores cambiaron. Entre 1945 y 1989, el énfasis fue evitar la expansión comunista, promover el desarme nuclear y mantener las esferas de influencia. A partir de 1989, los principales objetivos se han orientado a una mayor integración económica, la lucha en contra de las principales amenazas que prevalecieron a la Guerra Fría (terrorismo y narcotráfico), la búsqueda de un equilibrio financiero, el enfrentamiento al cambio climático, la reducción de la pobreza en el mundo, la defensa de los derechos humanos y la promoción de la democracia, entre otros.

El cambio de equilibrio del poder en el sistema internacional y los nuevos objetivos de política exterior de los principales actores fueron favorables para que otros actores, en este caso los gobiernos locales, pudieran aumentar su presencia e influencia en el sistema internacional. Anteriormente, en el marco del enfrentamiento Este-Oeste, los Estados nacionales se erigían como los principales actores en el ámbito global. Sus objetivos de política exterior más importantes estaban enfocados a cuestiones de seguridad y de mantenimiento de sus zonas de influencia. Por lo tanto, la política internacional se concentraba principalmente entre las superpotencias y sus respectivos aliados. Con esta lógica fuera del escenario y con la ayuda del proceso de globalización, los actores subnacionales empezaron a tener una mayor visibilidad en el contexto internacional. Desde esta perspectiva, los actores locales mexicanos buscan una mayor inserción en la nueva dinámica internacional para no aislarse de estos procesos y 
obtener beneficios. El aislamiento, en un ambiente globalizado, es altamente costoso para cualquier actor.

Por otro lado, la globalización económica y una mayor interdependencia han facilitado la presencia de los gobiernos locales mexicanos en actividades de naturaleza externa. Estos procesos han provocado que la competencia por mayores recursos entre los países y los actores locales haya aumentado significativamente. Es por ello que los estados y los municipios mexicanos buscan una mayor inserción externa para competir por esos recursos, como serían las inversiones externas, la transferencia tecnológica, las remesas, y las divisas que se generan por el turismo internacional y el comercio.

Asimismo, los procesos de integración económica y liberalización comercial que se han puesto de moda en los últimos años han contribuido a que los actores locales aumenten sus actividades de carácter externo. En la negociación de este tipo de acuerdos, las regiones y las localidades buscan también defender sus propios intereses y promover sus objetivos particulares. En el caso de México, la firma del Tratado de Libre Comercio con Estados Unidos y Canadá, que entró en vigor en 1994 (y otros acuerdos de naturaleza similar con diversos países), facilitó una mayor presencia de los estados y los municipios en el ámbito internacional. ${ }^{2}$ Además, la dinámica comercial y financiera que se ha desarrollado en los últimos años en la región del Asia Pacífico ha generado también incentivos para que los actores subnacionales mexicanos pongan atención en esa zona. Los estados mexicanos que colindan con la Cuenca del Pacífico han visto esa región como una opción para estrechar sus lazos externos.

Con la liberalización económica, los gobiernos locales pudieron, con mayor facilidad, colocar sus productos en los mercados internacionales y atraer inversión extranjera directa a sus territorios. Como se puede observar entonces, las transformaciones recientes en el sistema internacional y el cambio en los objetivos centrales de los actores han incentivado una mayor participación internacional de los

2 Sobre este punto, se recomienda: Fry (2014). 
gobiernos locales mexicanos, los cuales buscan evitar aislarse de las nuevas dinámicas globales con el objetivo de traer beneficios a las comunidades regionales.

\subsection{Segundo pilar: El modelo de desarrollo económico y el sistema político}

Al igual que el primer pilar, el contexto interno también explica el creciente interés de los gobiernos locales mexicanos por obtener una mayor inserción en la dinámica mundial. Los cambios políticos y económicos de las últimas décadas en el país son también responsables de que esos gobiernos hayan ampliado su margen de maniobra en este tipo de actividades. En primer lugar, es necesario mencionar que el proceso de apertura democrática ha impactado profundamente en la capacidad de maniobra de los gobiernos locales mexicanos. Anteriormente el sistema presidencialista no daba mucho margen de acción a los estados y municipios. Como el presidente prácticamente elegía a los candidatos a gobernadores y presidente municipales, éstos le debían lealtad al jefe del Ejecutivo y por lo tanto no competían con el gobierno federal en asuntos internacionales, puesto que la política exterior era prácticamente un monopolio del primer mandatario. En este contexto, los gobernadores estatales y presidentes municipales otorgaban una baja prioridad a los temas internacionales y, por lo tanto, preferían ocuparse de asuntos de política local y, en consecuencia, desarrollaban pocas actividades externas. Con la desaparición del régimen autoritario y centralista priista, los gobiernos locales tienen una mayor libertad de acción, lo que les ha permitido promover actividades hacia el exterior, sin la supervisión o un control estricto del gobierno federal.

A partir de 1989, cuando por primera vez un candidato a gobernador no priista ganó una elección estatal, los estados han aumentado su presencia en el ambiente internacional. Resulta lógico que un gobierno estatal de bandera partidista diferente al del federal busque desarrollar una mayor actividad externa porque cuenta con mayores márgenes de maniobra, pero también porque quiere mostrar una mayor independencia frente al presidente de la nación. En este sentido, los gobernadores han usado su presencia internacional como un mecanismo para guardar 
los equilibrios internos del sistema político mexicano. Esta alternancia en el poder local ha ampliado el margen de acción de las entidades federativas con el exterior. Anteriormente, era más difícil realizar todas esas actividades por la disciplina que mantenían los gobernadores de los estados frente a la figura presidencial. Hoy, esa realidad ha cambiado radicalmente. Este nuevo federalismo mexicano, con una mayor presencia internacional, busca proyectar una mayor división de poderes. Además, en los últimos años el gobierno federal ha impulsado políticas de descentralización administrativa, lo cual ha permitido a los gobiernos estatales tener mayores espacios de acción en otros temas de política nacional y, también, en cuestiones internacionales.

Las condiciones políticas, es decir, la ausencia o no de conflictos locales, también impacta en la intensidad de la presencia internacional de un actor local. Los estados con mayores problemas internos tienen una participación externa limitada, puesto que mucha de la atención y los recursos son utilizados para atender esos problemas. Algunos ejemplos son los estados de Oaxaca, Guerrero, Michoacán y Tamaulipas, entre otros. Estas entidades reflejan grandes desigualdades, problemas políticos y violencia producto de la guerra contra el narcotráfico. En los últimos años, estos estados se han mantenido relativamente aislado de los asuntos internacionales.

Los cambios económicos también han incentivado el fenómeno. En años anteriores, el modelo de desarrollo económico de México estaba basado en el esquema sustitutivo de importaciones. Una economía cerrada daba pocas oportunidades a los gobiernos locales para promoverse en el exterior. A partir de los años ochenta del siglo pasado, México inició un proceso de apertura económica caracterizado por la liberalización del comercio exterior y la atracción de inversiones externas. Con la nueva política económica, los gobiernos locales tuvieron una oportunidad de incrementar su presencia y visibilidad en los asuntos económicos exteriores. Ahora, estos actores buscan con mayor autonomía colocar sus productos locales en los mercados internacionales y atraer las inversiones externas con el fin de fomentar la creación de empleos y mejorar las condiciones socioeconómicas de sus comunidades. 
Existen también otros elementos internos que explican el interés de los gobiernos locales por participar en temas internacionales. Los más importantes son el desarrollo económico de la entidad, la posición geográfica y el tamaño del estado, y los rasgos demográficos, entre otros. En cuanto a las capacidades económicas, es obvio que las entidades de más alto desarrollo tendrán oportunidad de generar una mayor actividad internacional. Un producto interno elevado, mayores flujos de inversión, altas tasas de comercio exterior y mayores ingresos de divisas por turismo significan más recursos para financiar las actividades de carácter externo. En el caso de México, los estados más ricos son los que actualmente proyectan mayores actividades externas, como son el caso del DF, Nuevo León, Estado de México, Jalisco y Baja California. ${ }^{3}$ La única excepción es el caso de Chiapas, una entidad pobre en recursos financieros, pero muy activa en relaciones internacionales. En este caso, la intensidad se explica por factores coyunturales. En 1994, el surgimiento del movimiento zapatista elevó la visibilidad internacional de Chiapas. Ante esto, el gobierno local decidió impulsar una mayor actividad internacional para aprovechar esa coyuntura.

Otro factor que también determina la intensidad de internacionalización de los gobiernos locales es la posición geográfica y el tamaño de la entidad. Es claro que las entidades federativas mexicanas que comparten frontera con otros países tendrán una mayor participación. El compartir frontera implica, por necesidad, un contacto intenso con los vecinos. Igualmente, el tamaño de la entidad es relevante. Los estados mexicanos más grandes han desarrollado mayores labores de carácter externo, como Chihuahua, Coahuila y Baja California. En cambio, los estados más pequeños tienen una tendencia de menor participación, como son los casos de Tlaxcala, Aguascalientes, Colima, entre otros. Estos estados se han mantenido más aislados en comparación con sus pares y se han concentrado más en los temas locales. Una excepción es el caso del DF, una de las entidades más pequeñas, pero con una intensa labor externa. Al ser el centro político del país

\footnotetext{
Sobre una medición de las actividades internacionales de los estados mexicanos, se puede consultar: Jorge Schiavon (2006).
} 
y una entidad que recibe grandes flujos de inversión externa, el DF se ha posicionado como una de las ciudades con mayor actividad externa en los últimos años (Schiavon, 2006).

Las características demográficas de los estados o municipios también determinan en gran medida la intensidad y el tipo de participación internacional. El aumento de la migración de algunos estados de la federación y la permanencia de mexicanos en el extranjero, especialmente en Estados Unidos, han estimulado a los gobiernos estatales a mantener un contacto constante con esas comunidades y a abrir oficinas encargadas de su protección y atención. Las remesas que los mexicanos envían representan una fuente de financiamiento muy importante para impulsar el desarrollo de sus localidades. Por ello, los gobiernos estatales están poniendo especial atención en este fenómeno, buscando formas de facilitar el flujo de recursos e incentivando su uso en actividades productivas y no sólo de consumo. ${ }^{4}$

La estructura administrativa también determina la intensidad de actividades externas. Muchos estados y municipios mexicanos están abriendo oficinas de asuntos internacionales para la promoción de estas actividades. Asimismo, algunos están creando oficinas de representación en el extranjero. La existencia de estas estructuras administrativas permite ampliar el alcance de las actividades externas de los gobiernos locales mexicanos.

En el marco del contexto interno, otro elemento fundamental es el tipo de actores subnacionales que participan en este fenómeno. Uno de los actores más visible es el gobernador del estado o el presidente municipal. De hecho, la intensidad de la presencia internacional de las entidades federativas recae principalmente en estos dos actores. Para apoyar al ejecutivo local, existen varias secretarías estatales cuya función también tiene relación con lo externo. Por ejemplo, las secretarías de fomento económico de los estados desempeñan un papel importante en la proyección internacional del estado que

4 Sobre el tema de migración y paradiplomacia en el caso mexicano, se recomienda: Ortega \& Gallardo (2011, pp. 133-160). 
se trate. Las carteras estatales educativas, de seguridad, de turismo, medio ambiente y otras también desarrollan vínculos con el exterior. Es claro que estos temas despiertan interés entre los funcionarios de los gobiernos locales y tienen un amplio margen de maniobra para establecer cooperación internacional en estas áreas.

Otro actor gubernamental que empieza a hacerse visible en esta materia es la legislatura local. En algunos estados, los congresos estatales han creado comisiones que atienden los temas internacionales. Por ejemplo, Baja California tiene una comisión de asuntos fronterizos. Otro ejemplo es la Asamblea Legislativa del Distrito Federal, pues tiene una comisión de relaciones exteriores muy activa.

Existen otros actores no gubernamentales que también están involucrados estrechamente en estos asuntos. Por ejemplo, las empresas privadas desempeñan un papel importante para la proyección internacional de los estados y municipios. En algunos estados existen asociaciones de empresarios, las cuales tienen una intensa actividad con los asuntos externos. Asimismo, los partidos políticos locales se han convertido en participantes activos en estos temas. Los medios de comunicación también tienen una función especial en la proyección de la imagen internacional de las entidades. Los migrantes se han convertido en un actor al cual hay que ponerle atención. Las ONGs, principalmente de ayuda a migrantes, y las instituciones de educación superior también son actores clave en la materia.

Otros elementos fundamentales que se inscribe en el marco del contexto interno y que ayudan a explicar la creciente participación de los gobiernos locales mexicanos en asuntos internacionales son las motivaciones y objetivos que estos actores se plantean. En el caso mexicano, los gobiernos locales tienen diversas motivaciones para participar en asuntos internacionales. Las principales, pero no las únicas, son a) atraer inversiones al estado, b) promover los productos locales en los mercados internacionales, c) difundir los atractivos turísticos de la región para aumentar el número de divisas que llegan a la comunidad y d) fomentar la transferencia tecnológica. 
Los gobiernos locales mexicanos, cada vez en mayor medida, buscan atraer inversión extranjera para dinamizar la actividad productiva en su entidad y crear más empleos. Cada año, las autoridades realizan actividades de atracción, como son visitas al extranjero e invitaciones a empresarios foráneos para que conozcan la infraestructura, entre otras. Estas actividades se realizan de manera coordinada con la federación o en muchos casos de forma independiente. Además, como la competencia entre otras entidades mexicanas es mayor, muchas veces realizan esta labor de manera confidencial para que sus pares no se enteren y puedan obtener ellos las inversiones que se buscan.

Las entidades mexicanas buscan, también, mercados externos para colocar los productos locales. Todo ello con la finalidad de desarrollar mercados internos más competitivos y prósperos, así como para mejorar el bienestar en sus comunidades. Estas actividades las realizan a través del apoyo a empresarios locales para participar en ferias internacionales, con el objetivo de que se conozcan sus productos. Además, existen programas locales para apoyar la exportación de los productos más representativos, como sería el caso del tequila en Jalisco, el aguacate de Michoacán, el henequén en Yucatán, el tomate en Sinaloa, entre otros ejemplos. Inclusive, varios gobiernos locales han abierto oficinas en el exterior para promover los productos locales, como es el caso de Baja California que cuenta con oficinas en Estados Unidos y Europa.

Como el turismo se ha convertido en un motor importante de divisas, los estados con estos atractivos han desarrollado una labor intensa en el exterior para dar a conocer sus encantos. Muchos gobernadores han realizado visitas al exterior para hacer esta promoción o para invitar a empresarios extranjeros a invertir en desarrollos turísticos. Quintana Roo, Jalisco, Baja California Sur, Guerrero y Veracruz son estados que han impulsado estas actividades.

La generación de transferencia tecnológica también ha sido una importante motivación para que los gobiernos locales amplíen su alcance internacional. Como en el país no se genera tecnología de punta, los estados y municipios la tienen que buscar fuera de las fronteras. Para 
ello, muchos gobiernos han firmado acuerdos interinstitucionales o de hermanamiento para tener acceso a las nuevas tecnologías. Un ejemplo es el caso entre Jalisco y Alberta, en Canadá. A través de un acuerdo de hermanamiento, la provincia canadiense ha capacitado a ingenieros mexicanos en el área forestal.

A pesar de que las principales motivaciones son económicas, también existen objetivos de naturaleza cultural. Es claro que muchos gobiernos locales desean promover los rasgos culturales de su estado en los foros internacionales. Ello se hace para que los valores y costumbres locales sean conocidos más allá de las fronteras.

Finalmente, el marco legal local también determina el grado de participación. Hay casos en donde los gobiernos estatales tienen leyes que los obligan a tener una presencia externa importante. Por ejemplo, Nuevo León y Baja California tienen ordenamientos locales en el ámbito comercial, educativo y tecnológico, que promueven una activa vinculación con el exterior. En cambio, otros estados no tienen tan desarrollada su legislación en esta materia. En cuanto a la planeación en asuntos internacionales, hasta ahora los gobiernos locales mexicanos no han puesto mucha atención en este aspecto. En los planes y estrategias de gobierno locales, el tema internacional aparece aislado y no de forma explícita en la mayoría de los casos. Obviamente existen excepciones a esta regla. Por ejemplo, el estado de Nuevo León sí contempla en sus planes de desarrollo, de manera explícita, la actividad internacional como una prioridad de política pública. ${ }^{5}$ Otra entidad federativa que ha puesto mayor atención a la planeación de carácter internacional es Chiapas; este estado del sureste mexicano ha sido el primero en incluir los Objetivos de Desarrollo del Milenio en la Constitución local, lo cual representa un avance muy importante en la materia. ${ }^{6}$

\footnotetext{
5 Ver el documento Plan Estatal de Desarrollo 2010-2015, especialmente las páginas 112 y 113, del Consejo de Planeación y Evaluación del Estado de Nuevo León (2010).

6 Ver el documento $\mathrm{La}$ ONU en Chiapas: Fortaleciendo capacidades, especialmente en la página 5 de la ONU México (2013).
} 
En resumen, los cambios en el modelo de desarrollo económico y la apertura democrática en México han permitido una mayor participación de los gobiernos locales en asuntos internacionales. Por un lado, las principales motivaciones que tienen esos actores han sido de naturaleza económica. Por ello, una mayor liberalización de la economía mexicana les permite tener una mayor presencia en esos temas. Por el otro, los cambios que el sistema político experimentó y la descentralización administrativa ofrecen a los actores subnacionales mexicanos una mayor participación en el contexto externo.

\subsection{Tercer pilar: las percepciones y preferencias individuales}

La esfera individual también explica la creciente participación de los gobiernos locales mexicanos en asuntos globales. La visión y la percepción que tenga el ejecutivo local determinarán de manera fundamental el activismo internacional de la entidad. Si el gobernador o el presidente municipal consideran que lo externo es importante, entonces trataran de tener una presencia activa más allá de las fronteras. Pero si uno piensa que la actividad externa representa solamente un gasto sin beneficios tangibles, entonces la presencia externa se verá socavada. Es decir, los avances en este ámbito dependen de la voluntad del gobernador en turno: si se pone un mayor o menor énfasis en las relaciones del estado con el exterior, si se abren o se cierran oficinas y/o representaciones de asuntos internacionales, o si se otorga prioridad a ciertos temas, como comercio, migración, turismo, energéticos, entre otros. Esto conlleva a uno de los principales problemas de la presencia internacional de los gobiernos locales en México: la falta de institucionalidad y continuidad.

La personalidad y las ambiciones políticas del gobernante estatal son muy importantes. Por ejemplo, el Estado de México, bajo la administración de Enrique Peña Nieto (PRI), desarrolló un importante activismo político internacional, que rebasó las actividades económicas y comerciales de administraciones anteriores. Este activismo se debió, principalmente, a que el exgobernador tenía interés en ganar la candidatura a la presidencia bajo las siglas de su partido. El funcionario 
buscaba un mayor protagonismo político para hacerse más visible nacional e internacionalmente, y así lograr su ambición política.

Otro caso es el del Distrito Federal (DF). Cuando la capital del país fue gobernada por Andrés Manuel López Obrador (PRD), el DF tuvo una presencia internacional muy reducida. Este personaje afirmaba que la mejor política exterior era la política interna. Es significativo que el funcionario nunca realizó un viaje internacional durante su gestión. En el año 2006 llegó a la jefatura del DF Marcelo Ebrard, del mismo partido político, quien estudió Relaciones Internacionales en una prestigiosa universidad de México. Obviamente, Marcelo Ebrard tiene una visión muy distinta a su antecesor en materia de relaciones internacionales. Desde su llegada, el Distrito Federal ha intensificado considerablemente la presencia externa de su entidad.

Siendo así, con respecto a las características de los gobernadores que explican la participación internacional de su entidad, pareciera que, más que su filiación partidaria, es más importante su personalidad. Además de atraer inversión extranjera, promover las exportaciones locales y acercarse a las comunidades de sus emigrantes en el exterior, estos ejecutivos locales han aumentado considerablemente la actividad externa de sus entidades siguiendo una estrategia de diferenciación frente a otras entidades federativas y ante el propio ejecutivo federal, buscando un posicionamiento político en el ámbito nacional e internacional para avanzar su propia carrera. Generalmente buscan una mayor actividad internacional para legitimarse internamente $y$, de este modo, perfilarse a otros puestos de elección popular. Pero también hay quienes buscan mayores reflectores y protagonismo con estas actividades. Por ejemplo, muchos gobernadores y presidentes municipales buscan establecer acuerdos de hermanamiento con otras ciudades o firmar acuerdos solamente para fomentar su protagonismo.

Otras formas de prestigio político son las visitas que realizan los mismos gobernadores de los estados a otros países. Estas giras tienen igualmente el propósito de atraer inversiones extranjeras, promover el turismo del estado, promocionar los productos locales, firmar acuerdos de cooperación, visitar a la comunidad de paisanos, estre- 
char lazos culturales y, en general, ampliar la presencia del estado a escala internacional. Los destinos favoritos son Estados Unidos, algunos países de Europa, América Latina y algunos países de Asia. Pero también estos viajes se han hecho por intereses particulares de los gobernantes y presidentes municipales.

Asimismo, los gobernadores estatales y presidentes municipales reciben un número importante de visitantes extranjeros de fama internacional, principalmente representantes de organismos internacionales y de otros gobiernos, empresarios, artistas, premios Nóbel, entre otros, así como a los embajadores acreditados en México y a los cónsules ubicados en su propia entidad, con el fin de mejorar la relación entre el estado y sus países y atender asuntos de interés mutuo. En algunos casos, estas actividades se generan por las ambiciones políticas de los gobernadores. Por ejemplo, el jefe de gobierno del DF recibió entre 2006 y 2011 a un número importante de jefes de Estado y de gobierno del mundo. El objetivo era posicionarse para poder competir por la candidatura presidencial en 2012.

En resumen, los intereses personales de los funcionarios gubernamentales impactan de manera directa en la intensidad de actividades internacionales de estados y municipios. Las ambiciones y percepciones individuales pueden hacer una mayor o menor proyección internacional. Si un gobernador considera que los vínculos con el exterior son importantes, entonces es muy probable que la presencia de su estado en estas actividades sea mayor.

\section{El marco jurídico de la acción exterior de los gobiernos locales: El caso mexicano}

Tras el auge y desarrollo de las actividades externas de los gobiernos locales entrada la década de 1990, los gobiernos centrales mantuvieron una postura diferida frente al nuevo fenómeno que presenciaban al interior. Por un lado, había países que pretendían retener de manera celosa el monopolio de las relaciones internacionales. Por otro lado, figuraban naciones con posturas más flexibles, permitiendo, hasta cierto punto, la incursión de sus gobiernos locales en la esfera 
internacional. No obstante, por parte de los gobiernos centrales, a pesar de contar con posiciones contrarias, quedó entrevisto una característica común: varios países desarrollaron trabajos en materia legislativa encaminados a la regulación de los contactos que los gobiernos locales pretendían tener con sus contrapartes fuera de las fronteras nacionales.

Hoy, a más de veinte años de que los Estados en su mayoría emprendieron y aumentaron trabajos legislativos en aras de regular la actividad externa de los gobiernos locales, es difícil mantener un consenso global sobre las atribuciones que pueden ostentar. Incluso dentro de la comunidad de académicos dedicados a este tema se ha hecho hincapié sobre la necesidad materializar, paralelo al Derecho Internacional, una serie de normas que establezcan las pautas para la creación de un Derecho Local Internacional, con base en el derecho de la autonomía internacional de los gobiernos locales. Pese a la idea de coadyuvar esfuerzos entre las naciones para crear un marco legal globalmente aceptado, que encuadre la acción exterior de los gobiernos locales, es necesario recalcar que no se busca cuestionar el carácter exclusivo de las competencias del gobierno central en materia de relaciones internacionales y que, por el momento, se deja a la legislación nacional el poder de decidir la legitimidad de los actividades internacionales de las entidades federativas (Ciudad de México, Proyecto ALLAS, 2013, p. 51).

En México la situación no es diferente. Tanto a nivel federal, como local, se cuenta con normas establecidas en la Constitución Política que delimitan el quehacer de los gobiernos locales, tanto sus facultades como limitaciones. De acuerdo con el artículo 89, en su fracción X, "es facultad del ejecutivo dirigir la política exterior y celebrar tratados internacionales sometiéndolos a la aprobación del senado". Con esta norma, queda establecido el papel primordial del Estado frente a temas internacionales como también en la formalización de vínculos con sus contrapartes en el exterior a través de los tratados internacionales. Asimismo, el Senado figura como actor que regula la actividad exterior del Estado. Sus funciones van desde analizar la política exterior desarrollada por el Ejecutivo federal, aprobar los 
tratados internacionales (artículo 76, fracción 1), ratificar el nombramiento de agentes diplomático (artículo 76, fracción 11), hasta autorizar el permiso por ausencia del ejecutivo federal del territorio nacional siempre y cuando sea mayor a siete días (Art. 88).

Refiriéndose a los gobiernos locales, la constitución mexicana estipula un listado de facultades y/o zonas de injerencia en su artículo 115, así como también una serie de limitaciones en su artículo 117. Por ejemplo, el gobierno federal mantiene exclusividad en temas como seguridad nacional, defensa, tratados de libre comercio, entre otros. Por otra parte, los gobiernos locales pueden tener injerencia en temas tales como la protección del medio ambiente, atracción de inversiones, turismo, educación, deportes, entre otros. ${ }^{7}$

La situación en materia de regulación de la actividad internacional de los gobiernos locales en México había estado bajo el cobijo de la Constitución y las constantes modificaciones a la misma. No obstante, para 1992, y como resultado de trabajos legislativos, nace la Ley sobre la Celebración de Tratados (LCT), la cual permite a las dependencias u organismos descentralizados de la Administración Pública Federal, estatal o municipal, celebrar "acuerdos interinstitucionales"8 con uno $\mathrm{o}$ varios órganos extranjeros u organismos internacionales. El origen de esta ley puede ser derivado de dos fenómenos separados o por la combinación de ambos: por un lado, el auge y desarrollo de la participación de los gobiernos locales fuera de las fronteras nacionales y, por el otro, las rondas de negociaciones con miras a la consolidación de un tratado de libre comercio entre Canadá, Estados Unidos y México.

Posterior a la entrada en vigor de la Ley sobre la Celebración de Tratados, la tarea principal era, y es hasta el día de hoy, institucionalizar la acción exterior de los gobiernos locales mediante el asesoramiento directo de la Secretaría de Relaciones Exteriores. La idea con esto es

\footnotetext{
Sobre el marco legal para la acción internacional de los gobiernos locales mexicanos, se recomienda: Jorge A. Schiavon \& Rafael Velázquez (2008, pp. 39-54).

8 Convenio regido por el derecho internacional público, celebrado por escrito entre las autoridades previamente mencionadas.
} 
logar pasar de la informalidad a la formalidad, mediante los mecanismos de dictamen y registro de los acuerdos interinstitucionales (artículo 7 y 8). México, hasta este punto, había dado los primeros pasos encaminados a materializar un marco legal en donde la participación local en el exterior quedara regulada. No obstante, todavía falta mucho por recorrer para logar la meta que la Ley Sobre la Celebración de Tratados se propuso durante su concepción. El desconocimiento de dicha ley, la ambigüedad de la misma, además de la falta de mecanismos coercitivos que garanticen su cumplimiento, han sido las barreras a las cuales el gobierno federal, en específico la SRE y el departamento encargado de coordinar las acción exterior de los gobiernos locales al interior de la Secretaría se han enfrentado día con día.

En resumen, la actividad exterior de los gobiernos locales en México está inmersa en una zona gris, en donde el aumento de estas actividades ha rebasado por completo lo estipulado hace más de veinte años. En pocas palabras, tanto el crecimiento de la actividad exterior de los gobiernos locales, como los trabajos encaminados a regularla han crecido de manera desigual. Tomando en cuenta lo anterior, resulta indispensable reformar la Ley sobre la Celebración de Tratados, introduciendo mecanismos que encaminen a los titulares de las dependencias y al ejecutivo estatal o municipal a informar a la Dirección General de Coordinación Política sobre toda actividad que pretendan realizar más allá de las fronteras nacionales. Es fundamental contar con las estructuras institucionales de coordinación, tanto a nivel de los estados, como entre éstos y el gobierno federal, para asegurar que haya una política externa única y unificada de México frente al mundo pero que sea representativa de los intereses y preferencias de los gobiernos y actores locales (Schiavon, 2012, p. 106).

\section{Las relaciones internacionales de Baja California}

Baja California es una entidad con un perfil internacional por naturaleza. No solamente porque es una entidad fronteriza que tiene una intensa interacción con el vecino estado de California, sino también porque mantiene un vínculo estrecho con otras regiones del mundo. Particularmente, el estado ha desarrollado estrechos lazos con Asia, 
especialmente con China, Japón y Corea del Sur, países que cuentan con importantes inversiones en Tijuana y otras ciudades del estado. Asimismo, en los últimos años algunos países europeos han incrementado sus inversiones en Baja California. En la misma tónica, el flujo comercial que tiene la entidad con el mundo es muy intenso y ha crecido en los últimos años. Sus principales productos de exportación son pantallas planas de televisión, equipos médicos, vino, cerveza, autopartes, electrónicos, entre otros.

Por otro lado, en los últimos años el estado de Baja California ha tenido una proyección internacional de alto impacto. Por ejemplo, Tijuana es la única ciudad del país que cuenta con un vuelo directo a Shanghái, uno de los principales centros financieros y de comercio de Asia. Esta conexión posiciona a la entidad en lugar visible a escala global. Asimismo, en poco tiempo Tijuana tendrá el primer aeropuerto binacional en México. Es decir, ese puerto aéreo dará servicio tanto a la ciudad de Tijuana, como a la de San Diego al mismo tiempo. ${ }^{9}$ Este elemento facilitará los flujos migratorios y turísticos, y proyectará a la región decisivamente a nivel internacional. El aeropuerto binacional Tijuana/San Diego representa una factor de internacionalización muy destacado.

Baja California fue el primer estado gobernado por un partido de oposición en $1989 .{ }^{10}$ Ello le permitió a la entidad tener mayor independencia para involucrarse en temas internacionales. Anteriormente, los gobiernos estatales mexicanos no tenían una presencia importante en temas globales por el sistema presidencialista que existía. A partir de ese año, el estado empezó a tener una presencia importante en asuntos globales. Como resultado del cambio en la ideología política tras la llegada del PAN a Baja California, el manejo de los asuntos

\footnotetext{
9 Actualmente el aeropuerto de Tijuana colinda de manera directa con la franja fronteriza del condado de San Diego. Ambos gobiernos están construyendo un puente transfronterizo que conectará al aeropuerto con un complejo de edificios del otro lado de la frontera.

10 Desde los años treinta del siglo pasado, el Partido Revolucionario Institucional (PRI) ganó todas las elecciones estatales en el país. En 1989, el Partido Acción Nacional (PAN) obtuvo el triunfo en la gubernatura de la entidad.
} 
tanto internos como externos tuvo transformaciones notables. En este sentido, el gobernador ya no estaba sumido bajo el mandato del ejecutivo federal lo que conllevó a Baja California tener un mayor grado de autonomía y margen de maniobra en temas fundamentales. Derivado de lo anterior, el estado fronterizo experimentó un auge en temas internacionales aprovechando los beneficios de su privilegiada posición geográfica.

Cabe destacar que Baja California es una entidad internacional por naturaleza y no sólo por el hecho de compartir frontera con la primera economía del mundo, Estados Unidos; su presencia internacional se deriva de la conjunción de otros factores que, sumados, obtienen como resultado un estado con herramientas eficaces para insertarse en actividades internacionales con gran facilidad. Sólo por mencionar algunos datos, Baja California cuenta con uno de los puertos marítimos con mayor dinamismo en la costa del océano pacífico, a través del cual llega mercancía de gran parte del mundo, en su mayoría proveniente de Asia; además, este estado cuenta con tres aeropuertos internacionales y miles de kilómetros en carreteras y vías férreas, y con tres puertas de entrada (Tijuana, Mexicali y Tecate) a Estados Unidos, en donde la puerta entre Tijuana y San Ysidro se consolida como el punto de cruce fronterizo de personas más transitado e importante del mundo.

La dinámica internacional en Baja California resulta ser una de las más representativas del país. Según el Instituto Mexicano para la Competitividad (IMCO) en su informe titulado "Indice de Competitividad Estatal 2014: Las reformas y los estados", específicamente en el subíndice "aprovechamiento de las relaciones internacionales", el estado fronterizo bajo análisis ocupa el cuarto puesto, sólo detrás de Quintana Roo, Baja California Sur y Chihuahua. Al respecto, el informe utiliza en su mayoría variables económicas que van desde la balanza comercial del estado, Inversión Extranjera Directa (IED), flujo de turismo y los ingresos del mismo, hasta hospitales con certificación internacional. Bajo esta misma idea, cabe subrayar la diversidad de variables que pueden ser utilizadas para el análisis de las relaciones internacionales de los estados; no obstante, para efectos prácticos 
del informe, el IMCO sólo se basó en las actividades denominadas como "efecto" de otras llamadas "causa".

Las actividades denominadas como causa resultan de gran importancia, puesto que sus efectos suelen ser positivos, dependiendo de su buena ejecución. En este sentido, entiéndase como actividades causa aquellas como: la firma de acuerdos interinstitucionales (hermanamiento, de cooperación específica, cartas de intención, relación de amistad), giras internacionales, apertura de oficinas de representación y participación en ferias internacionales. Partiendo de lo anterior, el caso de Baja California resulta ser un caso interesante de estudio.

\subsection{Acuerdos interinstitucionales}

Retomando el término legal con origen en la Ley sobre la Celebración de Tratados de 1992, los acuerdos interinstitucionales aparecen en escena como una herramienta de vinculación con el exterior, la cual Baja California ha utilizado en múltiples ocasiones, en su mayoría con Estados Unidos. Según el Registro de Acuerdos Interinstitucionales (RAI) de la Secretaría de Relaciones Exteriores, Baja california ha formalizado los siguientes acuerdos:

Tabla 1. Acuerdos interinstitucionales de Baja California formalizados ante el RAI

\begin{tabular}{|l|l|l|l|l|}
\hline $\begin{array}{c}\text { Gobierno local } \\
\text { extranjero }\end{array}$ & \multicolumn{1}{|c|}{$\begin{array}{c}\text { País } \\
\text { extranjero }\end{array}$} & $\begin{array}{c}\text { Estado } \\
\text { mexicano }\end{array}$ & $\begin{array}{l}\text { Municipio/institu- } \\
\text { ción mexicana }\end{array}$ & \multicolumn{1}{|c|}{ Instrumento jurídico } \\
\hline California & $\begin{array}{l}\text { Estados } \\
\text { Unidos }\end{array}$ & $\begin{array}{l}\text { Baja } \\
\text { California } \\
\text { Gobierno del Estado, } \\
\text { Secretaría del Medio } \\
\text { Ambiente y Recursos } \\
\text { Naturales, Secretaría } \\
\text { de Educación Pública }\end{array}$ & $\begin{array}{l}\text { Acuerdo Interinstitucional } \\
\text { sobre Educación, Capacita- } \\
\text { ción Ambiental y Recursos } \\
\text { Naturales, suscrito el 30 de } \\
\text { Mayo de 2002. }\end{array}$ \\
\hline $\begin{array}{l}\text { Agencia de } \\
\text { Protección } \\
\text { California }\end{array}$ & $\begin{array}{l}\text { Estados } \\
\text { Unidos }\end{array}$ & $\begin{array}{l}\text { Baja } \\
\text { California }\end{array}$ & $\begin{array}{l}\text { Secretaría de Protec- } \\
\text { ción al Medio Am- } \\
\text { biente }\end{array}$ & $\begin{array}{l}\text { Memorando de Coopera- } \\
\text { ción para la Transferencia } \\
\text { del Equipo de Monitoreo } \\
\text { Atmosférico, suscrito el 15 } \\
\text { de Marzo de 2007. }\end{array}$ \\
\hline $\begin{array}{l}\text { Corte Superior } \\
\text { del Estado de } \\
\text { California }\end{array}$ & $\begin{array}{l}\text { Estados } \\
\text { Unidos }\end{array}$ & $\begin{array}{l}\text { Baja } \\
\text { California }\end{array}$ & Gobierno del Estado & $\begin{array}{l}\text { Acuerdo de Colaboración } \\
\text { suscrito el 30 de enero de } \\
\text { 2009. }\end{array}$ \\
\hline
\end{tabular}




\begin{tabular}{|c|c|c|c|c|}
\hline $\begin{array}{l}\text { Gobierno local } \\
\text { extranjero }\end{array}$ & $\begin{array}{c}\text { País } \\
\text { extranjero }\end{array}$ & $\begin{array}{c}\text { Estado } \\
\text { mexicano }\end{array}$ & $\begin{array}{l}\text { Municipio/institu- } \\
\text { ción mexicana }\end{array}$ & Instrumento jurídico \\
\hline $\begin{array}{l}\text { Embajada } \\
\text { Francesa }\end{array}$ & $\begin{array}{l}\text { República } \\
\text { Francesa }\end{array}$ & $\begin{array}{l}\text { Baja } \\
\text { California }\end{array}$ & Gobierno del Estado & $\begin{array}{l}\text { Acuerdo de Colaboración } \\
\text { firmado en la Ciudad de Ti- } \\
\text { juana, Baja California, el } 29 \\
\text { de octubre de } 2009 \text {. }\end{array}$ \\
\hline California & $\begin{array}{l}\text { Estados } \\
\text { Unidos }\end{array}$ & $\begin{array}{l}\text { Baja } \\
\text { California }\end{array}$ & Gobierno del Estado & $\begin{array}{l}\text { Memorándum de Enten- } \\
\text { dimiento firmado el } 3 \text { de } \\
\text { diciembre de } 2001 \text {. }\end{array}$ \\
\hline \multirow[t]{2}{*}{$\begin{array}{l}\text { Defensoría } \\
\text { Pública del } \\
\text { Condado de } \\
\text { San Diego }\end{array}$} & $\begin{array}{l}\text { Estados } \\
\text { Unidos }\end{array}$ & $\begin{array}{l}\text { Baja } \\
\text { California }\end{array}$ & Gobierno del Estado & $\begin{array}{l}\text { Acuerdo de Colaboración } \\
\text { dictaminado el } 17 \text { de febre- } \\
\text { ro de } 2015 .\end{array}$ \\
\hline & $\begin{array}{l}\text { Banco } \\
\text { Interame- } \\
\text { ricano de } \\
\text { Desarrollo }\end{array}$ & $\begin{array}{l}\text { Baja } \\
\text { California }\end{array}$ & $\begin{array}{l}\text { Secretaría de Finan- } \\
\text { zas }\end{array}$ & $\begin{array}{l}\text { Convenio firmado el } 22 \text { de } \\
\text { junio de } 2010 .\end{array}$ \\
\hline
\end{tabular}

Fuente: Dirección General de Coordinación Política (2015).

De manera oficial, Baja California sólo ha registrado 7 acuerdos ante el RAI; esto no significa que sean los únicos, puesto que bajo la situación de "no inscritos" hay alrededor de 15 acuerdos versados en diferentes temáticas con diferentes países. Lo mismo sucede con los municipios que integran el estado de Baja California, los cuales han caído en la misma dinámica, evadiendo lo estipulado en la Ley sobre la celebración de tratados, en su artículo 7, al no informar sobre la formalización de vínculos con sus contrapartes en el exterior.

Tabla 2. Acuerdos firmados por los municipios de Baja California

\begin{tabular}{|l|c|c|}
\hline \multicolumn{1}{|c|}{ Ciudad } & Total de acuerdos & $\begin{array}{c}\text { Total de acuerdos inscritos } \\
\text { en el RAI }\end{array}$ \\
\hline Tijuana & 14 & 4 \\
\hline Mexicali & 14 & 5 \\
\hline Tecate & 4 & - \\
\hline Playas de Rosarito & - & 1 \\
\hline Ensenada & - & - \\
\hline
\end{tabular}

Fuente: elaboración propia con datos de la Dirección General de Coordinación Política e informes de gobierno locales. 
Como se puede observar, Baja California presenta un número considerable de acuerdos (registrados y no registrados) con los cuales corrobora su alta participación en el exterior. No obstante, los acuerdos interinstitucionales son sólo una parte del total de variables a considerar, con el fin de abarcar todo lo relacionado a la acción exterior de este estado.

\subsection{Viajes y visitas}

Dentro de la agenda del actual gobernador, Francisco Vega de Lamadrid, se han concretado tres giras de trabajo al exterior en su casi año y medio de administración frente al gobierno del estado de Baja California. En su primera gira, el destino fue Estados Unidos. Durante su estancia en el país vecino, el gobernador recorrió Connecticut, Massachusetts y Washington, D.C., con el objetivo de promover el estado fronterizo, pero también tratar temas de derechos humanos y migración. En su segunda gira al exterior, el gobernador viajó a Europa, en concreto a España y Reino Unido. El objetivo general de su viaje era la promoción de Baja California, pero, en específico, del sector aeroespacial, en donde el gobernador lideró la delegación de su estado en el Farnborough International Airshow 2014, una de las más grandes exposiciones del sector aeronáutico y aeroespacial en el mundo, celebrado en Hampshire, Reino Unido. En su última gira, Francisco Vega viajó al continente asiático recorriendo Japón, Corea, Taiwán y Singapur con varios objetivos: retener y aumentar inversiones, promover el estado, y concretar nuevas inversiones. Durante su estancia, el gobernador mantuvo 32 reuniones de negocios, en las cuales consiguió como logro la promesa de inversión de 218.5 millones de dólares para la entidad fronteriza.

Paralelo a las giras del ejecutivo estatal, Baja California fue destino de más de 80 misiones exploratorias, desde organismos de promoción de diversos países, como Japan External Trade Organization, Hong Kong Investment y la Cámara de Comercio e Industria de Champagne-Ardenne, hasta empresas que buscan conocer las ventajas que 
presenta el Estado como destino de inversión. ${ }^{11} \mathrm{Al}$ respecto, queda al descubierto la importancia para el gobierno estatal el estrechar lazos con países de peso internacional con la meta primordial de promoción local en aras de atraer turismo e inversiones, y posicionar los productos locales en el exterior.

\subsection{Oficinas administrativas en temas internacionales}

Hoy el gobierno estatal cuenta con una oficina de Relaciones Internacionales que se ubica en la Secretaría de Turismo. ${ }^{12}$ Es decir, el gobierno ha buscado darle una perspectiva turística a las relaciones exteriores del estado. Esta oficina cuenta con representaciones en San Diego, Los Ángeles y Londres; en algún momento tuvo en China y Japón. Tener estas estructuras genera una mayor presencia internacional del estado, lo que permite incrementar las exportaciones de los productos bajacalifornianos y atrae mayores inversiones para promover la creación de empleos.

Toda actividad fuera de las fronteras nacionales debe ser planeada con el fin de consolidar una estrategia y cumplir con los objetivos planteados. En este sentido, aparecen 3 oficinas importantes en el proceso de internacionalización local: oficina de asuntos internacionales (OFAI), oficinas de representación en el exterior y consulados honorarios en el estado. En primera instancia, la OFAI se consolida como el área administrativa dentro de la administración pública local encargada de la coordinación, apoyo y gestión en temas de índole internacional.

\footnotetext{
11 Ver el documento 1er informe de gobierno del estado de Baja California 2013, eje 3, especialmente la página 125. Disponible en: http://www.bajacalifornia.gob.mx/informe/pdfs/ Eje $\% 203 \% 20$ Economia.pdf

12 Anteriormente, la oficina se encontraba adscrita a la Dirección de Relaciones Públicas, una dependencia ubicada en las oficinas del gobernador. Pero a partir de 2012, el gobierno estatal decretó su localización en la Secretaría de Turismo. Ver el documento en el Periódico Oficial: wnw.bajacalifornia.gob. $m x$ /portal/gobierno/legislacion/periodico/2012/junio/SECCI-29-06-2012.pdf
} 
En el caso de Baja California, la oficina encargada de esta tarea es la Dirección de Relaciones Internacionales, la cual se encuentra al bajo el esquema organizativo de la Secretaría de Turismo del estado (SECTURE). Al respecto, resulta indispensable incrementar la participación de esta oficina en las demás dependencias. Por ello, la Dirección General de Coordinación Política de la SRE ha emitido varios manuales para la creación de OFAIs, en donde destaca que dichas áreas administrativas deberían estar bajo la tutela del ejecutivo y con ello coordinar trabajos de manera horizontal con otras áreas del gobierno estatal. A pesar del bajo perfil con el que goza dicha oficina, lograron su adhesión a la Asociación Mexicana de Oficinas de Asuntos Internacionales de los Estados (AMAIE) en el año 2014.

Además de esto, Baja California, dentro de su estrategia de internacionalización, ha contemplado la apertura de oficinas de representación en el exterior, con el objetivo primordial de promover las ventajas que el estado ofrece. $\mathrm{Al}$ respecto, este estado cuenta con 3 oficinas en el exterior: una en San Diego, otra en Los Ángeles, Estados Unidos, y la última en Londres, Reino Unido. Otro indicador importante en el proceso de internacionalización es el número de consulados que se ubican en la entidad. En el caso de Baja California existen 22 consulados entre honorarios y generales en donde 13 son de origen europeo, 4 asiáticos, 1 africano, 2 de América latina y 2 de América del Norte.

\subsection{Turismo}

Una de las peculiaridades más grandes del Estado de Baja California es su reconocimiento internacional como destino turístico por parte de millones de personas al año. Datos de la SECTURE arrojan que la entidad recibió alrededor de 10 millones de turistas internacionales finalizado el 2013, siendo de origen hispano los que más frecuentaron la entidad seguido de turistas anglosajones. Paralelo a la llegada de turistas, la derrama económica producto de lo anterior tuvo un aumento considerable. Según datos de SECTURE, Baja California registró 80 millones de dólares sólo en el primer trimestre del 2015 con motivo del turismo. En palabras del subsecretario de turismo, 
Gabriel Lelevier, son números que no se habían registrado desde el 2007 (Martínez, 2015).

Pese a la percepción de un aumento en el flujo de turistas internacionales en el estado, la realidad es otra. A finales de 2013 se registraron alrededor de 10 millones de turistas, la misma cantidad que en 1992, mientras que al final de la década de los noventa, al igual que en el 2006-2007, la cifra rozó los 20 millones. La explicación a este fenómeno puede encontrarse en dos factores: la crisis internacional y el alza de la delincuencia en el estado de Baja California. En este escenario, la lógica dicta que a menor ingreso, menor será el poder adquisitivo de las personas para salir de viaje. Aunado a lo anterior, el alza de la delincuencia ha sido uno de los problemas que más ha afectado al turismo. Según el índice Incidencia delictiva del fuero común de la Secretaría de Gobernación, Baja California ha ocupado el primer lugar en sus últimos 3 informes anuales en cuanto a delincuencia, suceso que permea en toda actividad de promoción del estado. ${ }^{13}$

Pese a lo trágico que parece el impacto de la delincuencia en la actividad turística de Baja California, la situación muestra síntomas de cambio. Hoy en día, el estado experimenta un repunte en turismo con fines médicos. Según SECTURE, Baja California genera 40\% de la derrama económica por turismo de salud en México, lo cual ocasionó que se abriera la Dirección de Turismo de Salud, siendo la primera entidad del país que cuenta con una. Anualmente, Baja California capta alrededor de 500 millones de dólares por concepto de turismo de salud, al recibir entre 1.5 y 2 millones de pacientes en su mayoría hispanos (52\%) seguido de los anglosajones (37\%) (La razón, 2015).

\subsection{Integración de conglomerados económicos entre Baja California y California: Clúster aeroespacial y de equipos médicos}

Para una mayor internacionalización, el gobierno del estado de Baja California estableció una visión estratégica de largo plazo sobre el

\footnotetext{
13 Ver el documento, Incidencia delictiva del fuero común de la Secretaría de Gobernación, especialmente la página 5. Disponible en: bit.ly/1Jv0MKE
} 
desarrollo económico basada en la integración de conglomerados (clústeres). A partir de este modelo, la Secretaría de Desarrollo Económico (SEDECO) y el sector empresarial local se dieron a la tarea de identificar, diagnosticar e impulsar las vocaciones productivas y las nuevas áreas de oportunidad en la región. Estas medidas buscaban aprovechar las ventajas comparativas no solamente del estado de Baja California, sino de la región en su conjunto (California-Baja California) para integrar procesos productivos binacionales que pudieran generar beneficios económicos para ambos lados de la frontera. Los principales sectores que participan en este proceso son la industria turística, la electrónica, la agroindustria, la industria vitivinicultora, los dispositivos médicos, la cerveza, la industria aeroespacial, la automotriz, el software, la pesca y la acuacultura, entre otros. Estos sectores han experimentado un mayor dinamismo, debido a la perspectiva internacional que gobierno y empresas les han otorgado.

La dinámica industrial en Baja California ha traspasado barreras, llegando a tener gran influencia en California. Ambas entidades han venido experimentando procesos de integración en cuanto a técnicas de producción en el ramo médico y aeroespacial. Tan sólo en Tijuana, la industria de dispositivos médicos concentra alrededor de 60 plantas, mientras que la aeroespacial concentra 80, llevándose ambas el primer lugar nacional en cuanto a importancia y desempeño.

Referente al sector aeroespacial, éste recibió 185 millones de dólares de los cuales más de $50 \%$ provenía de inversiones estadounidenses. A su vez, Estados Unidos recibe la mayor parte de las exportaciones de productos derivados de este ramo. ${ }^{14}$ Con respecto al sector de equipos médicos, al igual que en el aeroespacial, más de la mitad de la inversión extranjera proviene de Estados Unidos. A su vez, Baja California exporta $90 \%$ de lo fabricado por este clúster a Estados unidos, en su mayoría a California, específicamente. El clúster de productos médicos ubicado en Baja California representa un caso

14 Ver el documento, "Industria Aeroespacial Mexicana Mapa De Ruta 2014” especialmente la página 41. Disponible en www.promexico.gob.mx/documentos/mapas-de-ruta/MRTAeroespacial-2014.pdf 
de éxito importante. Este clúster, en conjunto con el de San Diego, California, forma parte del agrupamiento binacional de productos médicos más diverso y sofisticado en Norteamérica. ${ }^{15}$

\subsection{Migración}

Baja California tiene una sociedad compuesta por migrantes; el fenómeno migratorio resulta natural para la población que siempre ha vivido en frontera. No obstante, el tema va más allá al analizar que la mayoría de la gente que arriba sólo pretende utilizar la entidad como trampolín hacia Estados Unidos. El problema surge cuando, al fracasar en el intento de conseguir el sueño americano, Baja California se convierte en su hogar, automáticamente. Aquí la problemática se convierte en política pública, puesto que hay que brindar cobijo, dentro de lo posible, a la comunidad de migrantes que quedó atrapada entre su sueño de mejorar su situación económica y un muro.

\subsection{Resultados en materia económica}

Tras el análisis de las actividades denominadas como "causa” llega el turno de mostrar los efectos y/o resultados que, de manera directa o indirecta, han producido en la economía local. Al respecto se tomarán 4 variables económicas para medir la efectividad de las "actividades causa": Inversión Extranjera Directa (IED), exportaciones, Producto Interno Bruto (PIB) y turismo.

\section{Inversión extranjera Directa}

En el marco de las giras internacionales del ejecutivo local, el objetivo primordial de su viaje estaba encaminado en la búsqueda de inversión para la entidad. Al respecto, Baja California mostró un aumento por más de 300 millones de dólares en cuanto IED. De acuerdo con los datos de la Secretaría de Economía, a finales de 2014 la cifra cerró

\footnotetext{
15 Ver el documento, "Sector Dispositivos Médicos en el mundo" especialmente la página 1. Disponible en: embamex.sre.gob.mx/espana/images/stories/d2013/10_Octubre/sector_dispositivos_medicos.pdf
} 
en los 813 millones de dólares en comparación con los 505 millones registrados un año anterior. La cifra final del 2014 representó a nivel nacional el 3.6\%, mientras que el origen de la IED quedó distribuido de la siguiente manera: Estados Unidos, 63.7\%, y Corea, 19.8\%, mientras que el otro $16.5 \%$ se repartió entre China y Japón. ${ }^{16}$

\section{Exportaciones}

Posterior a la llegada de la IED y de su puesta en marcha, el resultado de la producción de artículos especializados finaliza con su traslado; en pocas palabras, se hace alusión al proceso de exportación del producto elaborado según el origen del demandante. $\mathrm{Al}$ respecto, la Secretaría de Economía proyecta a Baja California hasta el 2013 en segundo lugar a nivel nacional con $9.9 \%$ del total de las exportaciones del país, acumulando un monto neto anual referente a esta actividad de 32’229,572 millones de dólares.

\section{Producto Interno Bruto}

Como resultado de la intensa dinámica comercial que caracteriza a Baja California tanto al interior, como exterior del estado, el PIB se convierte en un indicador importante sobre el valor total de la producción de bienes en la entidad. Al igual que la IED y las exportaciones, el PIB ha crecido de manera sostenida durante los últimos años. Retomando datos de la Secretaría de Economía, el PIB de Baja California en el 2013 se situó en 383.507 millones de pesos.

\section{Turismo}

Como parte de las actividades en el exterior con miras a la promoción del estado, mostrando sus bondades, el turismo tradicional parece no aumentar según los datos de la Secretaría de Turismo Estatal. No obstante, es de aplaudirse lo logrado respecto al turismo médico.

\footnotetext{
16 Ver el documento del Consejo Coordinador Empresarial de Tijuana, Análisis Económico de Tijuana 2014, especialmente la página 3. El documento se encuentra disponible en: www. cdt.org.mx/Portals/2/Documentos/indicadores/10.MAR.14_TIJUANA.pdf
} 
Hoy, Baja California es punto de referencia en este rubro llegando a considerarla dentro de la élite del turismo médico. La afluencia de personas dependerá en gran medida de lo realizado por el gobierno estatal en materia de promoción. Lo relacionado con seguridad pública deberá ser un trabajo en conjunto, puesto que, al aumentar la delincuencia, aumenta de igual manera la seguridad en la frontera, aspecto que desanima el cruce de personas hacia ambos lados, debido a las largas horas con motivo del reforzamiento de la seguridad. El mismo efecto se produce en las aduanas. En resumen, el alza en la delincuencia, como en la seguridad fronteriza, frena el comercio bilateral ocasionando pérdidas económicas para ambos lados de la frontera.

En resumen, Baja California ha consolidado una actividad internacional alta derivado de los cambios políticos que sufrió a fines de 1980, lo cual trajo consigo cierta libertad para expandir sus horizontes. Seguido de los cambios políticos, su expansión al área internacional nació como una necesidad, puesto que fenómenos como la globalización obligaron al estado a vincularse con otros países para atraer nuevas formas de financiamiento, en aras de mejorar la calidad de los servicios públicos, y como consecuente una mejora en la calidad de vida, a través de un crecimiento económico derivado de la atracción de IED, el posicionamiento de productos nacionales y promoción turística. Aunado a ello, la personalidad de los tomadores de decisiones fue trascendental, debido a que han sabido aprovechar las ventajas que Baja California posee, desde su posición geográfica hasta los rasgos demográficos que componen a la sociedad bajacaliforniana, una sociedad sustentada en la migración.

\section{Reflexiones finales}

La actividad internacional de los gobiernos subnacionales mexicanos experimentó una transformación importante a finales del siglo XX. Este trabajo ha dejado evidencia de que ese cambio se debió principalmente a las modificaciones en el sistema internacional y en el modelo de desarrollo económico y el sistema político mexicano. En el mismo orden de ideas, las ambiciones personales y las percepciones 
individuales por parte de los funcionarios locales han tenido un impacto significativo en dichas actividades. Por lo tanto, los principales pilares de la paradiplomacia mexicana son el equilibrio de poder del sistema internacional, el modelo de desarrollo económico, el funcionamiento del sistema político, así como los rasgos personales de los funcionarios de gobierno.

En el caso de Baja California, los pilares arriba mencionados también han incidido en una mayor internacionalización del estado. Por un lado, los cambios en el sistema internacional, la firma del TLCAN y un mayor dinamismo económico en el Asia Pacífico le han otorgado a la entidad un ambiente externo favorable para una mayor proyección internacional. Asimismo, los cambios en el modelo de desarrollo y la apertura democrática le permitieron un mayor margen de maniobra en esos temas. Finalmente, las percepciones e intereses personales de sus últimos gobernadores y presidentes municipales han permitido una mayor internacionalización del estado en los últimos años.

Todas las actividades descritas en los párrafos anteriores demuestran que Baja California ha sido uno de los estados del país con mayor proyección internacional de México. Si esta tendencia se mantiene, entonces la internacionalización de Baja California se fortalecerá en el futuro cercano. Existen actualmente las condiciones necesarias para una mayor internacionalización de Baja California. Existe la infraestructura económica necesaria, recursos humanos altamente capacitados, voluntad política, una ubicación geográfica privilegiada, estrechas relaciones con actores internacionales, entre otras ventajas.

Finalmente, este texto busca hacer algunas propuestas de política pública para una mayor internacionalización del estado. En primer lugar, no es mala idea darle un perfil turístico a la oficina de asuntos internacionales del gobierno estatal. Sin embargo, sería recomendable darle un perfil mayor. Por ejemplo, sería conveniente que la oficina se ubicara de manera directa en la oficina del gobernador. Así, el encargado de los asuntos internacionales se reportaría de manera directa con el gobernador y podría coordinar la actividad exterior de otras dependencias del gobierno estatal. También sería impor- 
tante reabrir las oficinas de representación en China y Japón para fortalecer los vínculos con la región. Otra recomendación es que la oficina de asuntos internacionales de Baja California mantenga una estrecha relación con la AMAIE para intercambiar experiencias con otras oficinas similares y poder tener retroalimentación que sirva para mejorar las funciones propias. Finalmente, es necesaria una mayor coordinación del estado con el gobierno federal, legisladores locales y federales, con organizaciones internacionales, con asociaciones de la sociedad civil, con empresarios, universidades y otros actores para una mayor internacionalización del estado.

\section{Referencias}

Aguirre, I. (2000). ¿Qué sentido tiene hablar de paradiplomacia? Una encuesta intertextual en torno a un neologismo polisémico. En F. Aldecoa \& M. Keating. Paradiplomacia: Las relaciones internacionales de las regiones, (pp. 203-235). Madrid: Marcial Pons.

Proyecto ALLAS. (2013). Marco legal e institucional para la acción internacional de las ciudades: diagnóstico y prospectiva euro-latinoamericana, (p. 51). México: Proyecto ALLAS.

Consejo Coordinador Empresarial de Tijuana. (2014). Análisis Económico de Tijuana 2014. Recuperado de: www.cdt.org.mx/Portals/2/Documentos/indicadores/10.MAR.14_TIJUANA.pdf

Consejo de Planeación y Evaluación del Estado de Nuevo León. (2010). Plan Estatal de Desarrollo 2010-2015. Recuperado de: http:/ / wmw.nl.gob. $m x /$ sites/default/files/genl_ped_2010_2015_0_1.pdf

Fry, E. H., (2014). The Development of Sub-State Cross-Border Interactions in North America 1994-2012: The Impact of NAFTA and Post-9/11 Security Policies. En The External Relations of Local Governments in North America after NAFTA: Trends and Perspectives, (pp.15-51). México: UABC.

Gobierno del estado de Baja California. (2014). 1er informe de gobierno del estado de Baja California 2014, eje 3, especialmente la página 125. Recuperado de: http://www.bajacalifornia.gob.mx/informe/pdfs/Eje\%203\%20 Economia.pdf

La Razón. (4 de febrero de 2015). Turismo de salud deja una derrama por 400 mdd en Baja California. Recuperado de: http://razon.com.mx/spip. php?article249565 
Martínez, G. (6 de abril de 2015). Captan 80 mdd por turismo en BC durante Semana Santa. Recuperado de: http:/ /jornadabc.mx/tijuana/06-04-2015/ captan-80-mdd-por-turismo-en-bc-durante-semana-santa

ONU México. (2013). La ONU en Chiapas: Fortaleciendo capacidades. Recuperado de: bttp:/ / bit.ly/V03ofy

Ortega Ramírez, A. S. \& Gallardo, P. Y. (2011). La emigración en las relaciones internacionales de los gobiernos estatales del centro de México. En M. Martínez (coord.), Estudios comparados de diplomacia local en las entidades federativas del centro de México, (pp. 133-160). México: UNAM.

Ortega Ramírez, A. S. (2012). Políticas migratorias sub-nacionales en México. Evaluación de las Oficinas Estatales de Atención a Migrantes. México: Plaza y Valdés.

PROMÉXICO. (2014). Industria Aeroespacial Mexicana Mapa De Ruta 2014. Recuperado de: http:/ /www.promexico.gob.mx/documentos/mapasde-ruta/MRT-Aeroespacial-2014.pdf

PROMÉXICO. (2013). Sector Dispositivos Médicos en el mundo. Recuperado de:embamex.sre.gob.mx/espana/images/stories/d2013/10_Octubre/sector_dispositivos_medicos.pdf

Schiavon, J. A. (2006). La proyección internacional de las entidades federativas: México ante el mundo. México: SRE.

Schiavon, J. A. (2012). Las relaciones internacionales de los gobiernos locales en México hacia 2012. En Schiavon. J., \& Velásquez, R. La política exterior de México 2012-2018: Diagnósticos y perspectivas, (pp. 101-106). México: Asociación Mexicana de Estudios Internacionales.

Secretaría de Gobernación. (2014). Incidencia Delictiva del Fuero Común de la Secretaría de Gobernación. Recuperado de: bit.ly/1Jv0MKE

Zeraoui, Z. (2011). Diplomacia paralela y las relaciones internacionales de las regiones, Desafíos, 23(I), 59-96. 
